\title{
ARCO BARRA DE ERICH OU PARAFUSO AUTORROSQUEANTE? QUAL É O MELHOR MÉTODO DE FIXAÇÃO MAXILO-MANDIBULAR? UMA REVISÃO DE LITERATURA
}

\author{
Juliana Barroso COELHO ${ }^{1}$ \\ Renata Ferreira XAVIER ${ }^{2}$ \\ Cássio Roberto Rocha dos SANTOS 3 \\ Saulo Gabriel Moreira FALCI ${ }^{4}$
}

Recebido em: 21/05/2013 - Aprovado em: 30/07/2013 - Disponibilizado em: 15/08/2013

RESUMO: O objetivo dessa revisão da literatura foi determinar, entre Arco Barra de Erich e Parafuso autorrosqueante, qual é o melhor método de fixação maxilo-mandibular. A busca bibliográfica foi realizada por meio da Biblioteca Virtual em Saúde (BVS), nas seguintes bases de dados: LILACS (Literatura Latino Americana e do Caribe em Ciências da Saúde), SciELO (ScientificElectronic Library Online), MEDLINE (Literatura Internacional em Ciências da Saúde) e COCHRANE (Cochrane Library), IBECS e BIREME, até maio de 2012. Foram utilizadas as seguintes palavras-chave e as combinações dessas palavras no idioma Inglês: Intermaxillary Fixation, Intermaxillary Screws Fixation, Maxillomandibular Fixation, Maxillomandibular Screws Fixation, Erich Arch Bar, Erich Arch Bars, Mandibular fracture repair e Maxillofacial fracture fixation. Foram incluídos artigos relacionados com a fixação intermaxilar com a utilização de arcos barras de Erich e parafusos corticais. Foram analisados os artigos em seres humanos em que esses métodos foram avaliados juntos ou separadamente e do total de artigos 98 foram lidos na íntegra. Após a leitura, foram incluídos 20 artigos e excluídos 78 por não preencherem os critérios de inclusão. Observou-se que arco barras de Erich é o método mais utilizado pela maioria dos cirurgiões bucomaxilofaciais, no entanto, apesar de efetivo apresenta desvantagens. Como alternativa, os parafusos autorrosqueantes tem demonstrado eficiência e consideráveis vantagens. Entretanto, para que esse método de fixação maxilo-mandibular seja implementado com segurança, é necessário que haja mais estudos comparando as duas técnicas, pois durante a revisão da literatura, foi encontrado apenas um ensaio clínico comparando-as.

Palavras chave: Fixação maxilo-mandibular. Fixação intermaxilar. Arco barras de Erich. Parafusos autorrosqueantes. Fraturas mandibulares.

\footnotetext{
${ }^{1}$ Acadêmica do curso de graduação em Odontologia, Universidade Federal dos Vales do Jequitinhonha e Mucuri, Diamantina, Minas Gerais, Brasil. - julianabarrosoc@ yahoo.com.br

2 Acadêmica do curso de graduação em Odontologia, Universidade Federal dos Vales do Jequitinhonha e Mucuri, Diamantina, Minas Gerais, Brasil. - renatafxavier@yahoo.com.br

${ }^{3}$ Cirurgião Dentista, Especialista em Mestre em Cirurgia Bucomaxilofacial (PUC-RS), Doutor em Patologia Bucal (FOB-USP), Professor Titular de Cirurgia Bucomaxilofacial (UFVJM). - cassiorocha3@ gmail.com

4 Cirurgião Dentista, Especialista em Cirurgia Bucomaxilofacial, Mestre em Clínica Odontológica (UFVJM), Doutorando em Clínica Odontológica - CTBMF (FOP-Unicamp). - saulofalci@ hotmail.com
}

ABSTRACT: The purpose of this literature review was to determine, among Erich Arch Bar Bolt autorrosqueante, what is the best method of maxillo-mandibular fixation. A literature search was performed using the Virtual Health 570 
Library (VHL), the following databases: LILACS (Latin American and Caribbean Health Sciences), SciELO (ScientificElectronic Online Library), MEDLINE (from the International Literature health) and Cochrane (Cochrane Library), and IBECS BIREME until May 2012. We used the following keywords and combinations of those words in the English language: Intermaxillary Fixation, Fixation Screws Intermaxillary, Maxillomandibular Fixation, Fixation Screws Maxillomandibular, Erich Arch Bar, Erich Arch Bar, Mandibular fracture repair and Maxillofacial fracture fixation. We included articles related to intermaxillary fixation with using Erich arch bars and cortical screws. We analyzed the papers in humans in which these methods were evaluated together or separately and the total 98 articles were read in full. After reading, 20 articles were included and 78 excluded for not meeting the inclusion criteria. It was observed that Erich arch bars is the most common method used by most maxillofacial surgeons, however, has its disadvantages though effective. Alternatively, bolts and autorrosqueantes has shown significant efficiency advantages. However, for this method of fixing maxillomandibular be deployed safely, it is necessary that further studies comparing the two techniques, because during the literature review, we found only one trial comparing them.

Keywords: maxillo-mandibular fixation. Intermaxillary fixation. Erich arch bars. Autorrosqueantes screws. Mandibular fractures.

\section{Introdução}

Existem várias técnicas de fixação maxilo-mandibular em cirurgia bucomaxilofacial descritas na literatura. Entre elas se destacam: corrente elástica ortodôntica (SMITH, 1993); dispositivo de fixação rápida, sem uso de fios metálicos (PIGADAS et al., 2008); parafuso autoperfurante (GIBBONS; HODDER, 2003); fios Dimac (AYOUB; ROWSON, 2003); arco barra colado com resinas (CHANDAN; RAMANOJAM, 2010) e arco barra de Erich fixado através de fios de aço flexível.

Ao longo dos anos, estudos têm sido realizados com intenção de melhorar o método de bloqueio maxilo-mandibular (ARTHUR e BERARDO, 1989, RAI, DATARKAR e BORLE, 2011, IMAZAWA et al., 2006, VARTANIAN e ALVI, 2000,
HASHEMI e PARHIZ, 2011), reduzindo as taxas de complicações e maximizando os resultados. O desenvolvimento do parafuso autoperfurante, proporcionou aos cirurgiões bucomaxilofaciais diminuição no tempo operatório, rapidez e redução do risco de perfuração das luvas durante o ato operatório. Além disso, quando comparado com os arcos barra de Erich não se observa dano ao tecido periodontal e melhor manutenção da saúde gengival (JONES, 1999).

Apesar de em algumas situações clínicas específicas, alguns autores relatarem que a fixação maxilo-mandibular é desnecessária (FORDYCE et al., 1999), essa ainda é aplicada na maioria dos procedimentos bucomaxilofaciais em que se deseja alcançar uma oclusão estável. Nos dias atuais, a tomada de decisão clínica, deve ser 
baseada em evidência científica e testada com metodologias apropriadas através de estudos prospectivos e ensaios clínicos randomizados. Além disso, a qualidade dos estudos deve ser avaliada de forma minuciosa, para que se possa adotar uma prática clínica confiável onde se espera obter um resultado satisfatório no tratamento dos pacientes. Assim, o objetivo desse trabalho foi verificar através de uma revisão da literatura qual o melhor método de fixação maxilo-mandibular entre os arcos barras de Erich e parafusos corticais, descrevendo-se os aspectos positivos e negativos de cada técnica.

\section{Metodologia}

Antes de iniciar o estudo, os critérios de inclusão e exclusão foram definidos pelos autores. Foram incluídos no estudo artigos relacionados com a fixação intermaxilar com a utilização de $\operatorname{arcos}$ barras de Erich e parafusos corticais para fixação intermaxilar. Foram analisados os artigos em seres humanos em que esses métodos de fixação intermaxilar foram avaliados juntos ou separadamente. Os desenhos de estudos incluídos foram: ensaios clínicos randomizados, ensaios clínicos, estudos prospectivos, estudos transversais e estudos retrospectivos.

Foram excluídos da análise aqueles artigos que apresentavam outros métodos de fixação intermaxilar que não fossem arco barra de Erich ou parafusos corticais, ou que o objetivo principal não fosse a avaliação do método de fixação. Foram excluídos também aqueles artigos de revisão sistemática ou revisão da literatura, casos clínicos, relatos de casos, séries de casos, cartas ao editor, comentários, notas técnicas e aqueles artigos que não apresentavam resumos.

A revisão da literatura foi realizada a partir de uma busca eletrônica por meio da Biblioteca Virtual em Saúde (BVS), nas seguintes bases de dados: LILACS (Literatura Latino Americana e do Caribe em Ciências da Saúde), SciELO (Scientific Electronic Library Online), MEDLINE (Literatura Internacional em Ciências da Saúde) e COCHRANE (Cochrane Library), IBECS e BIREME.

Para a realização da pesquisa foram utilizadas as seguintes palavras-chave e as combinações dessas palavras no idioma Inglês: Intermaxillary Fixation, Intermaxillary Screws Fixation, Maxillomandibular Fixation, Maxillomandibular Screws Fixation, Erich Arch Bars, Erich Arch Bar, Mandibular fracture repair e Maxillofacial fracture fixation.

A primeira busca de artigos foi feita em março de 2012, e a última em maio do mesmo ano. $\mathrm{Na}$ primeira análise com a avaliação dos resumos e títulos, do total de artigos encontrados, 98 foram selecionados e lidos na íntegra. Após a leitura, foram 
incluídos 20 artigos na revisão da literatura e excluídos 78 por não preencherem os critérios de inclusão.

\section{Resultados}

\section{Arco Barra de Erich}

Vários autores têm investigado arcos barra de Erich como uma técnica de fixação maxilo-mandibular. Terris, Lalekea e Tuffo (1994) destacam que esse bloqueio maxilomandibular é utilizado em $68 \%$ dos casos das fraturas de mandíbula, sendo a colocação de arcos barra de Erich a primeira escolha da maioria dos cirurgiões bucomaxilofaciais.

Coletti, Salama e Caccamese (2007) afirmaram que arco barra de Erich é um método comprovado para fixação e são úteis para aproximar e imobilizar segmentos de fraturas cominutivas. Por outro lado, Ansari et al. (2011) afirmam que seu uso é contraindicado em indivíduos com coroas extensas, trabalho de ponte, indivíduos desdentados e fraturas mandibulares em crianças; sendo que esta última é devido aos dentes decíduos possuírem um cíngulo que podem causar avulsão dos fios circundantes utilizados para fixação.

Não se pode ignorar os benefícios dos arcos barra de Erich. Segundo Brindley (1988), esse tipo de fixação apresenta grande número de ganchos para a tração elástica, permitindo, com isso, a distribuição equilibrada da tensão elástica sobre o arco. Além disso, Imazawa et al. (2006) observaram que em casos de emergência, em que muitas vezes é necessário a rápida remoção dos fios, o dispositivo de arco de barras de Erich serve como uma tala para o osso fraturado.

Apesar dos arcos barra de Erich fornecerem um meio eficaz e versátil para fixação intermaxilar, seu uso inclui consequências como declararam Hashemi e Parhiz (2011), tais como, risco de penetração o que causa prejuízo para o cirurgião, trauma para o periodonto e comprometimento da higiene oral. Laurentjoye et al. (2009) realizaram um estudo e afirmaram que a aplicação dos $\operatorname{arcos}$ barra de Erich é demorada, sua remoção é difícil, e os doentes tem fala e alimentação dificultadas. Neste mesmo estudo declararam também que quando um fio é torcido em torno de um dente ele é sujeito à tração e há perigo de avulsão se a força for muito grande. Outro importante inconveniente citado por Arthur e Berardo (1989) causado por essa técnica, é o risco que o cirurgião-dentista corre de ser infectado por HIV e outras doenças infecciosas ao perfurar sua luva durante o procedimento.

\section{Parafuso autorrosqueante}

O uso de parafusos intraorais para fixação intermaxilar, como uma alternativa 
aos arcos barra de Erich, foi proposto em 1989 por Arthur e Berardo (1989).

Em seu estudo Schneider et al. (2000) relataram que parafusos autorrosqueantes são indicados para a fixação de fraturas em pacientes desdentados, com uma adequada prótese para permitir uma oclusão estável, e para fraturas mandibulares localizadas na sínfise, no corpo, no terço médio e no ângulo. Roccia et al. (2005) em seu estudo também indica esse método para a fixação de fraturas simples ou duplas com deslocamento mínimo. Por outro lado Vartanian e Alvi (2000) concordam que o uso de parafusos é contraindicado em pacientes com fraturas cominutivas ou múltiplas fraturas mandibulares com segmentos flutuantes na região anterior do arco. Em contrapartida Laurentjoye et al. (2009) contraindicam o uso dessa técnica em pacientes pediátricos com dentes retidos.

Os benefícios dos parafusos autorrosqueantes são relatadas por vários autores. Imazawa et al. (2006) em seu estudo declararam que os parafusos não só restauram a oclusão dentária adequada e função temporomandibular natural comum, como também oferecem várias vantagens. Hashemi e Parhiz (2011) afirmaram que a técnica é de rápida, segura e fácil inserção; a remoção é fácil e indolor; em ambulatórios, sem anestesia; e a saúde gengival e a higiene dental são mais fáceis de manter. Além disso, reduz o risco do cirurgião perfurar luvas e pele, evitando a transmissão de doenças infectocontagiosas como descreveram Arthur e Berardo (1989), Hashemi e Parhiz (2011), Imazawa et al. (2006) e Laurentjoye et al. (2009).

Com relação às desvantagens da utilização dos parafusos para fixação maxilomandibular, poucos são os relatos. Um estudo realizado por Rai, Datarkar e Borle (2011) mostrou que as complicações ocorridas no pós-operatório foram a quebra e afrouxamento do parafuso e cobertura da cabeça deste pela mucosa. A lesão iatrogênica de raízes no momento da inserção, que pode ser minimizada por um experiente cirurgião, foi outra complicação observada por Hashemi e Parhiz (2011).

\section{Comparação entre Arco Barra de Erich e Parafusos autorrosqueantes}

Comparando com arcos barra de Erich, os parafusos autorrosqueantes tem sido exaltados pelos cirurgiões de trauma por causa da sua favorável velocidade e perfil de segurança, segundo Ansari et al. (2011). No estudo realizado por Vartanian e Alvi (2000) foi observado que o tempo médio de inserção dos parafusos foi 15 minutos em comparação com 45 a 100 minutos requeridos para fixar os arcos barra de Erich, o que economiza uma quantidade significativa de tempo e custo.

Em um ensaio clínico randomizado realizado por Rai, Datarkar e Borle (2011) foi 
concluído que quando comparado ao arco barra de Erich, os pacientes que foram tratados com parafusos apresentaram uma melhor manutenção da higiene oral e menos complicações. Nesse estudo, 60 pacientes foram tratados com parafusos (grupo I) e 30 pacientes com arco barra de Erich (grupo II); como resultado, no grupo II a incidência de perfuração de luvas foi $36,66 \%$ e a incidência de trauma nos dedos dos operadores $18,18 \%$, enquanto que nenhuma dessas complicações foi vista no grupo I.

Em um estudo realizado por Monte et al. (2008) com 62 pacientes e 272 parafusos, analisados ao longo de um período de quatro anos, embora tenha havido complicações, a sua incidência foi baixa; o que foi comprovado também por Roccia et al. (2005) em um estudo que avaliou quarenta pacientes e obteve como resultado a principal complicação: perda de parafusos em 4,4\% dos casos, seguido pela cobertura de mucosa oral em 1,2\% dos casos. No entanto, nenhum dano à raiz, quebra do parafuso, má oclusão ou má consolidação da fratura foi observado. Esse mesmo autor preconiza que a experiência e habilidade manual do cirurgião na perfuração do osso, que é menos denso que a raiz dentária, pode minimizar riscos iatrogênicos.

No caso de uma emergência, comparando os dois métodos de fixação, o acesso à cavidade oral é mais fácil quando se utiliza parafuso, sendo muitas vezes necessário cortar mais de dez fios em pacientes com arco barra de Erich, enquanto apenas dois fios tem de ser cortados para os pacientes utilizando parafusos abrir a boca, de acordo Imazawa et al. (2006).

\section{Considerações Finais}

Arco barra de Erich é o método de fixação maxilomandibular mais utilizado pela maioria dos cirurgiões bucomaxilofaciais. Esse método, apesar de efetivo apresenta desvantagens como trauma para o periodonto, comprometimento da higiene oral, risco de perfuração de luva podendo acarretar contaminação com doenças infectocontagiosas, demorada inserção e difícil remoção. Como alternativa a esse método, os parafusos autorrosqueantes tem demonstrado eficiência e consideráveis vantagens, sendo uma técnica rápida, segura e de fácil inserção, a remoção é fácil e indolor, tem risco reduzido de perfuração de luvas e a saúde gengival e a higiene dental são fáceis de manter. Entretanto, para que esse método de fixação maxilo-mandibular seja implementado com segurança, é necessário que haja mais estudos comparando as duas técnicas, pois durante a revisão da literatura, foi encontrado apenas 1 ensaio clínico comparando-as. 


\section{Referências}

1. ANSARI, K.; HAMLAR, D.; HO, V.; HILGER, P.; CÔTÉ, D.; AZIZ, T. A comparison of anterior vs posterior isolated mandible fractures treated with intermaxillary fixation screws. Arch Facial Plast Surg., v. 13, n. 4, p. 266-70, jul-aug., 2011.

2. ARTHUR, G.; BERARDO, N. A simplified technique of maxillomandibular fixation. J Oral Maxillofac Surg., v. 47, p. 1234, 1989.

3. AYOUB, A.F.; ROWSON, J. Comparative assessment of two methods used for interdental immobilization. J Craniomaxillofac Surg., v. 31, n. 3, p. 159-61, jun., 2003.

\section{BRINDLEY, H. P. Maxillofacial} fracture fixation, prostheses, methods and dences. In: ALLING III, C. C.; OSBON, D. B. Maxillofacial Trauma. Philadelphia: Lea e Febiger., p. 195-223, 1988.

5. CHANDAN, S.; RAMANOJAM, S. Comparative evaluation of the resin bonded arch bar versus conventional erich arch bar for intermaxillary fixation. J Maxillofac Oral Surg., v. 9, n. 3, p. 231-5, sep., 2010.

6. COLETTI, D.P.; SALAMA, A.; CACCAMESE, J.F. Application of intermaxillary fixation srews in maxillofacial trauma. J Oral Maxillofac Surg., v. 65, n. 9, p. 174650, sep., 2007.

7. FORDYCE, A.M.; LALANI, Z.; SONGRA, A.K.; HILDRETH, A.J.; CARTON, A.T.; HAWKESFORD, J.E. Intermaxillary fixation is not usually necessary to reduce mandibular fractures. Br J Oral Maxillofac Surg., v. 37, n. 1, p. 52-7, feb., 1999.

8. GIBBONS, A.J.; HODDER, S.C. A self-drilling intermaxillary fixation screw. $\mathbf{B r} \mathbf{J}$ Oral Maxillofac Surg., v. 41, n. 1, p. 48-9, feb., 2003.

9. HASHEMI, H.M.; PARHIZ, A. Complications using intermaxillary fixation screws. J Oral Maxillofac 
Surg., v. 69, n. 5, p. 1411-4, may., 2011.

10. IMAZAWA, T.; KOMURO, Y.; INOUE, M.; YANAI, A. Mandibular fractures treated with maxillomandibular fixation screws (MMFS method). J Craniofac Surg., v. 17, n. 3, p. 522-9, may., 2006.

11. JONES, D.C. The intermaxillary screw: a dedicated bicortical bone screw for temporary intermaxillary fixation. Br J Oral Maxillofac Surg., v. 37, n. 2, p. 115-6, apr., 1999.

\section{LAURENTJOYE，M.; MAJOUFRE-} LEFEBVRE, C.; SIBERCHICOTi, F.; RICARD, A.S. Result of maxillomandibular fixation using intraoral cortical bone screws for condylar fractures of the mandible. J Oral Maxillofac Surg., v. 67, n. 4, p. 767-70, apr., 2009.

13. MONTE, J.; GONZÁLEZ-LAGUNA, J.; BUEN, J.; MADRID, J. A.; MARTÍ, G. Complications of selftapping bone screws for maxillomandibular fixation in the treatment of jaw fracture. Rev. Esp.
Cir. Oral maxilofac., v.30, n. 4, p. 265-269, ago., 2008.

14. PIGADAS, N.; WHITLEY, S.P.; ROBERTS, S.A.; MCALISTER, K.; AMEERALLY, P.; AVERY, C.M. A randomized controlled trial on crossinfection control in maxillofacial trauma surgery: a comparison of intermaxillary fixation techniques. Int J Oral Maxillofac Surg., v. 37, n. 8, p. 716-22, aug., 2008.

15. RAI, A.; DATARKAR, A.; BORLE, R.M. Are maxillomandibular fixation screws a better option than Erich arch bars in achieving maxillomandibular fixation? A randomized clinical study. J Oral Maxillofac Surg., v. 69, n. 12, p. 3015-8, dec., 2011.

16. ROCCIA, F.; TAVOLACCINI, A.; DELL'ACQUA, A.; FASOLIS, M. An audit of mandibular fractures treated by intermaxillary fixation using intraoral cortical bone screws. J Craniomaxillofac Surf., v. 33, n. 4, p. 251-4, aug., 2005.

17. SCHNEIDER, A.M.; DAVID, L.R.; DEFRANZO, A.J.; MARKS, M.W.; MOLNAR, .J.A; ARGENTA, L.C. 
Use of specialized bone screws for intermaxillary fixation. Ann Plast

Surg., v. 44, n. 2, p. 154-7, feb., 2000.

18. SMITH, A.T. The use of orthodontic chain elastic for temporary intermaxillary fixation. Br J Oral

Maxillofac Surg., v. 31, n. 4, p. 2501, aug., 1993.

19. TERRIS, D.; LALEKEA, L.; TUFFO, K. Mandibular fracture repair: specific indication for newer techniques.

Otolaryngol Head Neck Surg., v.

111, n. 6, p. 751-757, 1994.

20. VARTANIAN, A.J.; ALVI, A. Bonescrews mandible fixation: an intraoperative to arch bars. Otolaryngol Head Neck Surg., v. 123, n. 6, p. 718-21, dec., 2000. 